\title{
Estudio, mediante microscopía, del proceso de microfisuración de un hormigón sometido a compresiones previas
}

\begin{abstract}
Jose manuel Fernandez Paris, Prof. Num. de Física Jefe del Laboratorio de Ensayos Químicos de INTEMAC. (Instituto Técnico de Materiales y Construcciones)
\end{abstract}

\section{N T R O D U C C I O N}

Para apreciar la acción física de microfisuración que experimentan los hormigones, cuando se encuentran sometidos a esfuerzos de compresión, se emplean en la actualidad diversas técnicas, siendo las más utilizadas la prospección radiográfica, los métodos fotoelásticos, y la observación mediante microscopía.

La prospección radiográfica presupone la enorme ventaja de suministrar información tridimensional y en un tiempo muy breve; pero no permite una percepción lo suficientemente nítida de las fisuras como para establecer una clasificación e identificación de las mismas.

Las técnicas fotoelásticas, que fueron las que se utilizaron en los comienzos de este tipo de estudios, se han abandonado ya que, únicamente, ofrecen una información muy limitada y restringida a observaciones bidimensionales e, incluso, se hace muy difícil la perfecta identificación de las fisuras.

La observación mediante microscopía presenta dos grandes inconvenientes: requiere una elevada preparación técnica y la observación de las probetas es lenta y laboriosa.

Puede considerarse como una técnica destructiva, lo que supone la eliminación de la muestra analizada. No obstante, por otra parte, tiene la ventaja primordial de poder observar de una forma nítida, en determinadas condiciones de trabajo, cualquier tipo de fisura $y$, por tanto, permite su recuento y clasificación.

Los investigadores SANGHA y DHIR (1) comenzaron a estudiar el comportamiento del hormigón bajo carga, y estos mismos autores comprobaron posteriormente que la acción de las cargas originaba microfisuras, que podían observarse mediante microscopía (2), recogiendo las ideas desarrolladas por MEYER (3).

Comunicación presentada en la IX Asamblea Técnica Nacional de Hormigón Pretensado, formando parte del trabajo denominado INFLUENCIA DE LOS ESTADOS PREVIOS DE CARGA DEL HORMIGON SOBRE SU MICROFISURACION Y RESISTENCIA debido a J. Calavera Ruiz, A. Delibes Liniers, J. M. Izquierdo y Bernaldo de Quirós, y J. M. Fdez. Paris. 
Para este tipo de investigación es preciso adoptar una técnica que reduzca al mínimo las posibilidades de distorsionar las distintas fases estructurales que integran el material objeto de análisis.

Los conocimientos de la Microscopía Petrográfica actual permiten dos técnicas o metodologías: La observación mediante luz transmitida y mediante luz reflejada.

Las técnicas de luz transmitida requieren preparar láminas muy delgadas, aproximadamente de 63 micras del material que se observa, lo cual es prácticamente imposible de conseguir a partir de morteros y hormigones.

La técnica de luz reflejada, usualmente empleada en Metalografía y en la observación del clínker de cemento portland (5), requiere un esmerado proceso de pulido para que la superficie obtenida sea lo más especular posible y refleje la máxima cantidad de luz durante la observación.

Las razones aducidas motivaron la elección de esta última técnica, que evidentemente iba a permitir la identificación de las microfisuras. Cabe pensar que la adopción de esta técnica implicaría información muy limitada, ya que aparentemente se restringe, como en los métodos fotoelásticos, a un campo de observación bidimensional. A este respecto hay que indicar, principalmente, a efectos de validez del recuento de fisuras, que en la técnica cuantitativa de la microscopía por luz reflejada, la ley de HOLMES y CHARLES (6) y (7) relaciona el área observada durante el recuento y la fracción verdadera del componente. Siempre que dicha relación se cumpla, los resultados pueden considerarse como procedentes de un espacio tridimensional.

En el presente trabajo se pretende establecer una correlación entre la génesis y propagación de las microfisuras en el hormigón y los estados previos de carga a que dicho material ha sido sometido.

Es un hecho conocido (2), (8) que el hormigón presenta, o puede presentar, una microfisuración inicial en la superficie de contacto entre la pasta de cemento hidratado y los áridos, que tiene lugar antes de estar sometido el hormigón a la acción de cargas externas e, incluso, los investigadores citados han comprobado que si la relación "distancia entre partículas de árido/radio del árido" es inferior a 0,2, lo que se verifica en la generalidad de los hormigones, aparecen tensiones elevadas en la interfase "pasta de cemento hidratado/áridos" que dan lugar al comienzo de un proceso de fisuración. Tales fisuras son propias de fenómenos de retracción, asentamiento de los áridos y reflujo del agua de amasado.

Independientemente de estas fisuras, en la zona inferior de los áridos, debido a la compactación del hormigón, se forman burbujas de aire, ya que cada partícula de árido actúa de barrera impidiendo la expulsión del aire; estas burbujas debilitan la adherencia, pero no deben considerarse de origen tensional.

Los investigadores ALEXANDER y WAROLAW (9) han comprobado que las fisuras no suelen producirse en la superficie de los granos de arena y que dependen, fundamentalmente, del tamaño máximo del árido, en el sentido de que la tensión de rotura de adherencia aumenta cuando disminuye el tamaño máximo.

Otros autores, como MOAVENZADEH y BREMER (10), indican que las fisuras se producen generalmente en los áridos inclinados $45^{\circ}$ con la dirección de la carga, en los áridos lajosos o planos y en las zonas de segregación del árido grueso.

Con los datos expuestos como premisas y los resultados logrados por los investigadores CALAVERA, GONZALEZ VALLE y GONZALEZ ISABEL (11) se han realizado los trabajos del presente estudio. 


\section{PLAN DE ENSAYOS}

Se fabricaron doce pilares de hormigón de $0,30 \times 0,30 \times 1,80 \mathrm{~m}^{3}$ con la siguiente dosificación:

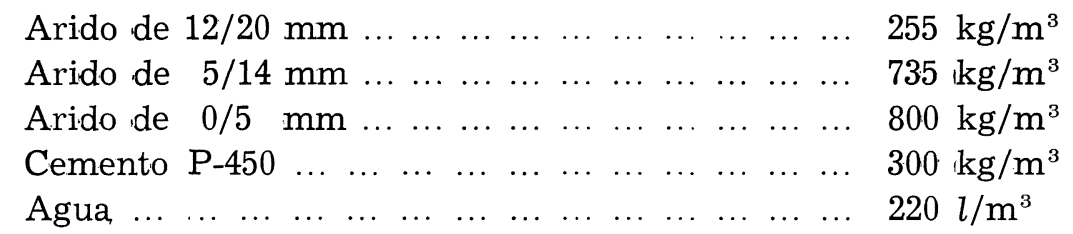

Los pilares se mantuvieron enmoldados, cubiertos con una arpillera húmeda y protegidos por láminas de material plástico durante un período comprendido entre $48 \mathrm{~h} \mathrm{y} 72 \mathrm{~h}$. El curado posterior se efectuó en una cámara acondicionada a $60 \% \mathrm{HR}$ y $20^{\circ} \pm 2^{\circ} \mathrm{C}$ de temperatura, hasta el día anterior a su ensayo, que se disponían en las condiciones ambientales del laboratorio.

Dos pilares se ensayaron a velocidad constante $\left(5 \mathrm{kp} / \mathrm{cm}^{2} / \mathrm{s}\right)$, hasta su rotura, que se produjo a $225 \mathrm{kp} / \mathrm{cm}^{2}$.

Dos pilares se sometieron durante 4 horas al $60 \%$ de la carga de rotura prevista y otras tres parejas, durante el mismo tiempo, al 70,75 y $80 \%$ respectivamente de la carga de rotura estimada con los dos primeros.

Los dos pilares sometidos al $80 \%$ de la carga de rotura se rompieron durante el proceso de mantenimiento. No obstante, se extrajeron testigos de las zonas menos afectadas.

\section{METODO OPERATORIO}

\section{Extracción de los testigos y preparación de las muestras}

De cada uno de los pilares, una vez sometidos al tratamiento indicado se extrajeron, mediante sonda de corona de diamante, tres testigos de $10 \mathrm{~cm}$ de diámetro y $30 \mathrm{~cm}$ de altura.

De cada probeta-testigo se cortaron dos lajas para su examen por microscopía, mediante una sierra de disco de diamante.

Por consiguiente, se observaron 6 lajas para cada escalón de carga experimentado.

Cada una de las lajas se sometía a un proceso de pulido y tinción que comprendía las fases siguientes:

- 60 minutos con CSi grado n. 120 Buehler,

- 60 minutos con CSi grado n. 240 Buehler,

- 90 minutos con CSi grado n. ${ }^{\circ} 600$ Buehler;

como vehículo de pulido se empleó agua destilada.

En cada cambio de abrasivo la superficie se sometía a la acción de un dardo de agua, para eliminar los residuos acumulados en los poros y fisuras.

Una vez terminado el proceso de pulido se seca la superficie mediante aire caliente.

Se impregna la zona pulida con una disolución de Rojo Bengala al $1 \%$ en etanol, mediante un pincel suave, y se deja secar durante 30 minutos. 
Posteriormente se eliminaba el colorante en exceso mediante un pulido muy leve, durante 2 minutos, con CSi n..$^{\circ} 600$, y se trataba con un dardo de etanol, con la finalidad de suprimir el abrasivo. Se dejaba secar durante 60 minutos.

\section{Observación al microscopio}

Las lajas, una vez pulidas y coloreadas, se marcaban mediante una plantilla (figura 1) con la finalidad de efectuar un barrido uniforme de las seis franjas interiores. El anillo externo de $2 \mathrm{~cm}$ de ancho no se tenía en consideración (fotografía 1) debido a que el hormigón podría haber experimentado efectos distorsionales ocasionados por la sonda de extracción.

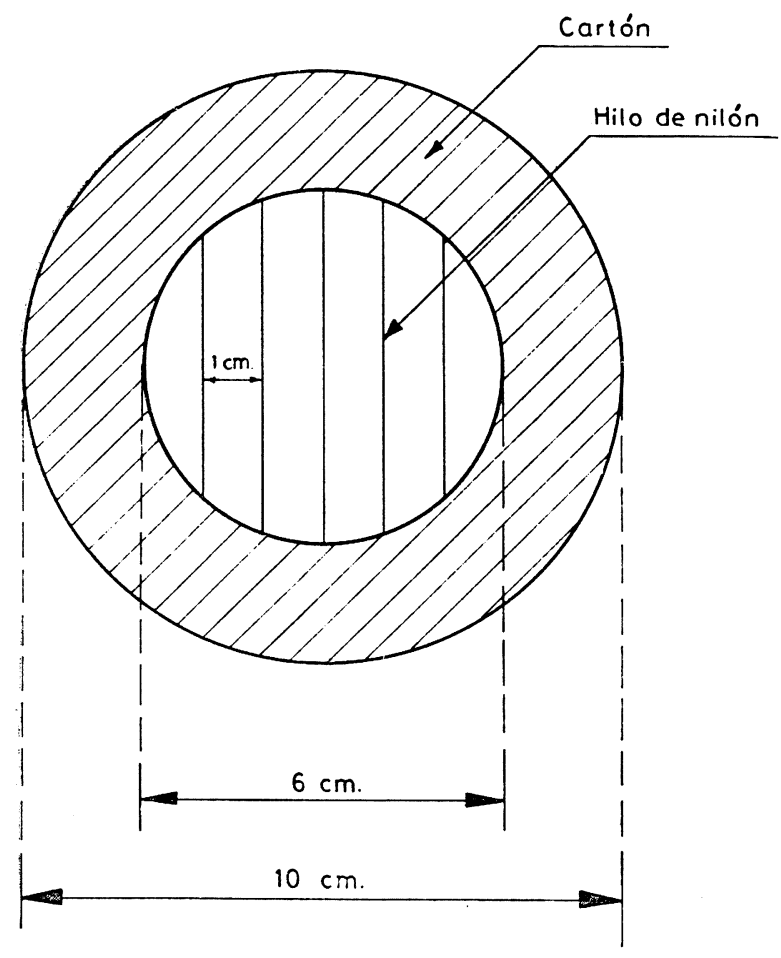

Fig. 1.-Plantilla para recuento de la preparación.

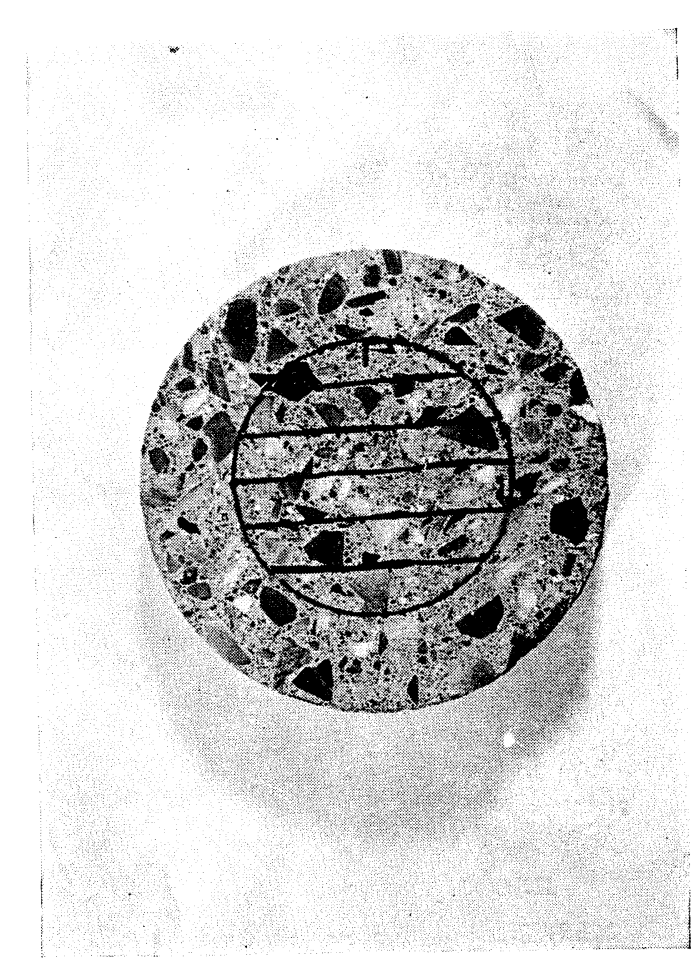

Foto 1.-Preparación dispuesta para el recuento de fisuras.

La laja, así dispuesta, se colocaba sobre la platina de una lupa binocular y se observaba con luz oblicua a $45^{\circ}$ y 20 aumentos, para el recuento de las fisuras. La apreciación de los detalles ocasionales se realizaba a 30 y 40 aumentos.

\section{Clasificación de los tipos de fisuras}

Para su recuento las fisuras se han clasificado en fisuras de adherencia y fisuras de mortero.

\section{Fisuras de adherencia}

Se originan entre los áridos gruesos y el mortero de cemento - Tipo 1 de la fig. 2- y se forman antes de estar sometido el hormigón a la acción de las cargas externas. Se deben a fenómenos de asentamiento, retracción y flujo de agua de amasado. 


\section{Fisuras de mortero}

Se forman en el seno de la matriz del mortero, y se subdividen en:

- Fisuras abiertas, aquellas que uno de sus extremos está unido a una fisura de adherencia y el otro extremo pertenece a la pasta del mortero. Tipo 2.1 de la fig. 2.

- Fisuras cerradas, aquellas que sus dos extremos se encuentran en la matriz de mortero. Tipo 2.2 de la fig. 2.

Fig. 2.-Identificación de los tipos de fisuras.

$$
\begin{array}{r}
\text { Tipo 1._Fisuras de adnerencia. } \\
\text { Tipo 2.-Fisuras de mortero. } \\
\text { 2.1.-Fisuras abiertas. } \\
\text { 2.2.-Fisuras cerradas. }
\end{array}
$$

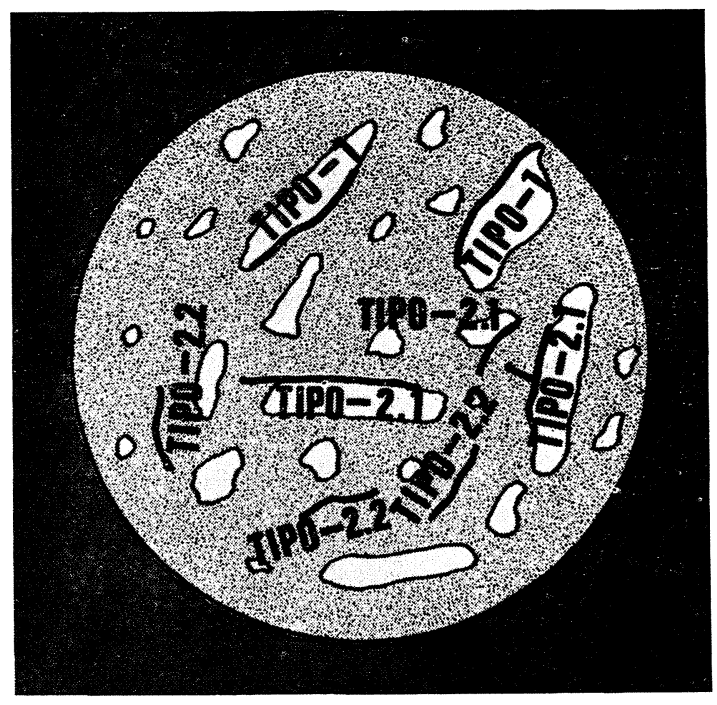

Las fisuras de mortero se detectan, según SHU (8), en aquellos hormigones que presentan una reducción de su capacidad al comportamiento bajo carga.

\section{RESULTADOS DE LA OBSERVACION}

Operando conforme se ha indicado se obtuvieron los siguientes resultados, que para su

\begin{tabular}{|c|c|c|c|c|c|c|}
\hline \multirow{3}{*}{ SERIE } & \multirow{3}{*}{ PILAR } & \multicolumn{4}{|c|}{ FISURAS OBSERVADAS } & \multirow{3}{*}{ TOTAL } \\
\hline & & \multirow{2}{*}{ ADHERENCIA } & \multicolumn{3}{|c|}{ DE MORTERO } & \\
\hline & & & Cerradas & Abiertas & TOTAL & \\
\hline \multirow{2}{*}{$\begin{array}{c}\text { S- } 0 \\
\text { CARGA } \\
0 \%\end{array}$} & $\begin{array}{l}P_{1} \\
P_{2}\end{array}$ & $\begin{array}{l}24 \\
25\end{array}$ & $\begin{array}{l}2 \\
1\end{array}$ & $\begin{array}{l}2 \\
2\end{array}$ & $\begin{array}{l}4 \\
3\end{array}$ & \\
\hline & MEDIA & 25 & & & 4 & 29 \\
\hline \multirow{2}{*}{$\begin{array}{c}\text { S-1 } \\
\text { CARGA } \\
60 \%\end{array}$} & $\begin{array}{l}P_{1} \\
P_{2}\end{array}$ & $\begin{array}{l}32 \\
21\end{array}$ & $\begin{array}{l}3 \\
4\end{array}$ & $\begin{array}{l}31 \\
62\end{array}$ & $\begin{array}{l}34 \\
66\end{array}$ & \\
\hline & MEDIA & 27 & & & 50 & 77 \\
\hline \multirow{2}{*}{$\begin{array}{r}\text { S-2 } \\
\text { CARGA } \\
70 \%\end{array}$} & $\begin{array}{l}P_{1} \\
P_{2}\end{array}$ & $\begin{array}{l}20 \\
16\end{array}$ & $\begin{array}{l}7 \\
2\end{array}$ & $\begin{array}{l}54 \\
65\end{array}$ & $\begin{array}{l}61 \\
67\end{array}$ & \\
\hline & MEDIA & 18 & & & 64 & 82 \\
\hline \multirow{2}{*}{$\begin{array}{c}S-3 \\
\text { CARGA } \\
75 \%\end{array}$} & $\begin{array}{l}P_{1} \\
P_{2}\end{array}$ & $\begin{array}{l}17 \\
18\end{array}$ & $\begin{array}{l}3 \\
4\end{array}$ & $\begin{array}{l}73 \\
66\end{array}$ & $\begin{array}{l}76 \\
70\end{array}$ & \\
\hline & MEDIA & 18 & & & 73 & 91 \\
\hline \multirow{2}{*}{$\begin{array}{c}\mathrm{S}-4 \\
\text { CARGA } \\
80 \%\end{array}$} & $P_{1}$ & 23 & 4 & 61 & 65 & \\
\hline & MEDIA & 23 & & & 65 & 88 \\
\hline
\end{tabular}
más fácil comprensión se han dispuesto en la gráfica 1.

Gráfica 1.-Resultado del examen por microscopía. 
En las probetas de los pilares no sometidos a carga se observa un predominio de fisuras de adherencia (fotos 3 y 4 ).

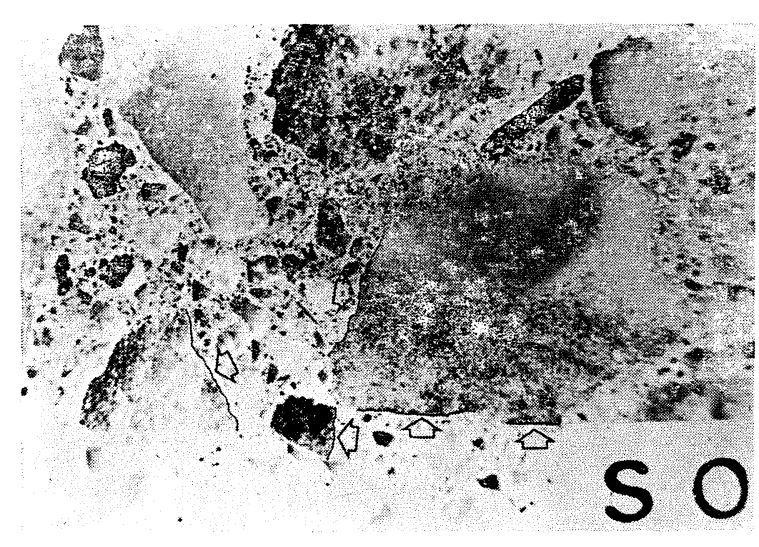

Foto 3.-Fisuras de adherencia en hormigón no sometido a compresiones previas.-10 $x$.

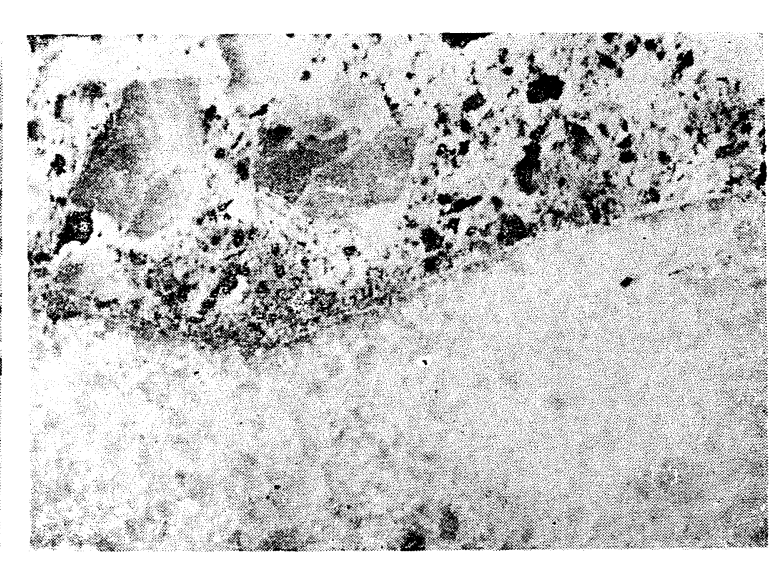

Foto 4-Detalle de una fisura de adherencia en hormigón sin esfuerzo previo.-25 $\times$.

Los testigos de los pilares sometidos a un $60 \%$ manifiestan un aumento de fisuras de adherencia y un elevado número de fisuras de mortero (foto 5).

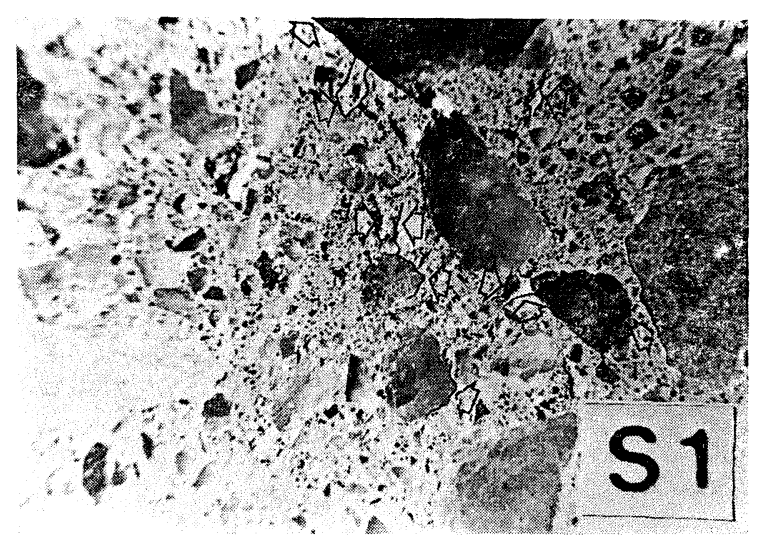

Foto 5.-_Fisuras de adherencia y de mortero en un hormigón sometido a una carga del $60 \% .-10 \times$.
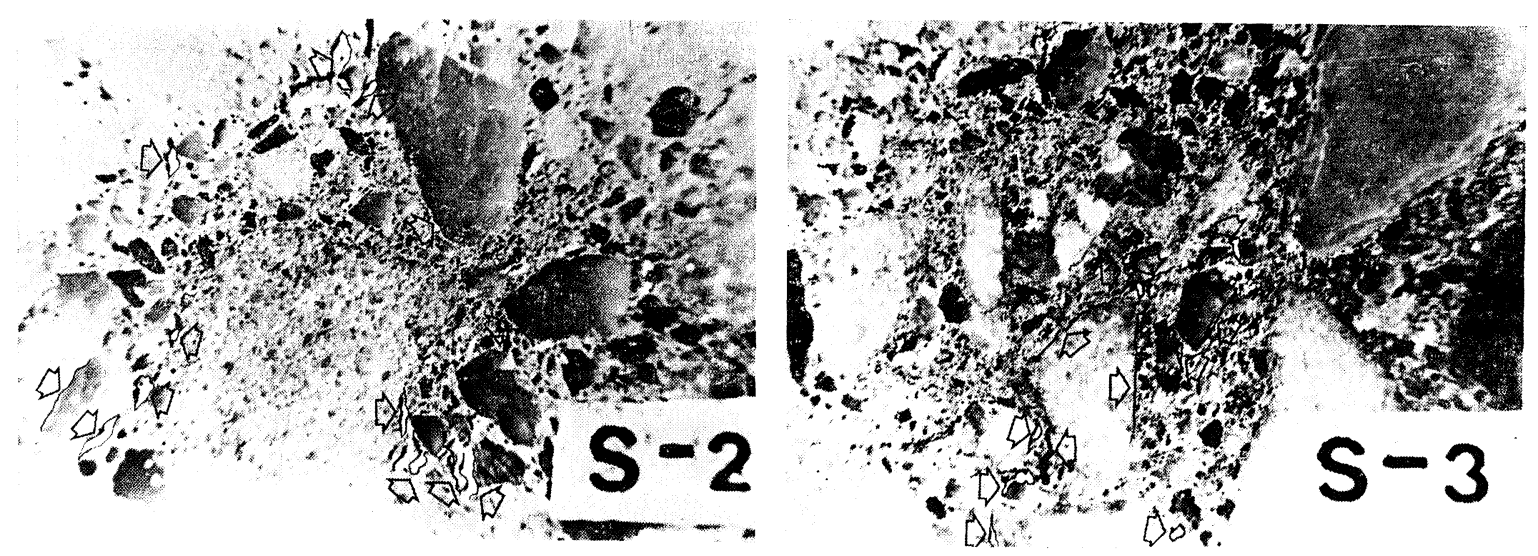

Foto 6.-Fisuras de mortero, cerradas y abiertas, en un Foto 7 .-Fisuras de mortero en un hormigón sometido hormigón sometido a una carga del ro $\% .-10 \times$. al $75 \% .-10 \times$.

60 
Las observaciones sobre testigos sometidos a un $70 \%$ y un $75 \%$ muestran que las fisuras de adherencia disminuyen, mientras que aumentan ostensiblemente las abiertas de mortero. Este hecho parece indicar que la fisuración abierta del mortero se debe en parte a las fisuras de adherencia. Por consiguiente debe interpretarse que parte de las fisuras de adherencia quedan encubiertas como fisuras de mortero abiertas (fotos 6 y 7).

En estos mismos testigos se aprecian zonas en que el árido se ha disgregado en la pasta cementante, observándose al microscopio como el efecto producido por una perdigonada sobre el material.

Los testigos sometidos a una carga del $80 \%$ de la rotura presentan amplias zonas con fuerte disgregación del material, lo que produce una observación muy dificultosa e imposibilita el recuento de las fisuras.

\section{O N C L U S I O N E S}

El método de observación al microscopio permite identificar y clasificar las fisuras en el seno del hormigón.

De acuerdo con MEYER (3) las fisuras de mortero, y principalmente las denominadas abiertas, motivan la reducción en la capacidad del hormigón al comportamiento bajo carga.

A partir de un $60 \%$ de su carga de rotura, comienzan a formarse fisuras de mortero que se originan tomando como base las de adherencia, ya existentes, o las formadas hasta alcanzar el $60 \%$ de su carga.

A la vista de lo observado en este estudio y de acuerdo con los trabajos de SLATE (12) y SHU (8) el proceso de microfisuración de un hormigón sometido a compresiones previas podría interpretarse de la manera siguiente:

- En todos los hormigones existen unas fisuras de adherencia en la interfase "pasta/áridos", debidas al asentamiento de los áridos, a las tensiones de retracción originadas durante el proceso de secado, y al flujo del agua de amasado.

- Hasta compresiones del orden del $60 \%$ de la carga de rotura se forman nuevas fisuras de adherencia y aumenta la longitud y la anchura de las ya existentes.

- A partir del $70 \%$ de la carga de rotura se originan fisuras de mortero abiertas y una gran cantidad de fisuras cerradas, que en determinadas zonas aisladas producen disgregaciones denominadas "efecto perdigonada".

Finalmente puede indicarse que la observación mediante la técnica de microscopía de luz reflejada aplicada al hormigón indica si ha estado o no sometido previamente a cargas elevadas, si bien es difícil precisar en qué grado sufrió el hormigón dicha compresión.

\section{B I B L I O G R A F I A}

(1) C. M. SANGHA y R. K. DhiR: Materiaux et Constructions. (1972).

(2) R. K. DhIR y C. M. SANGHA: Materiaux et Constructions. (1974).

(3) B. L. MEYER: A.C.I. Journal. (1969).

(4) Deljalkin, Lapin e Iwanow: Técnicas Petrográficas. Veb. Verlag Technick Berlín. (1960).

(5) J. M. Fernandez Paris: Microscopía del Clínker de Cemento Portland. Publicaciones del Instituto Eduardo Torroja. (1968).

(6) A. H. Holmes: Petrographic Methods and Calculations. Ed. Murby. London (1927).

(7) Chayes: Petrographic Modal Analysis. Ed. J. Wiley. New York (1956).

(8) T. T. C. SHU: A.C.I. Journal. (1963).

(9) K. M. AleXander y J. Warolaw: Natura. (1960).

(10) F. Moavenzadeh y T. W. Bremmer: Conf. Structure Solid (1969).

(11) J. Calavera, E. Gonzalez Valle, y G. Gonzalez Isabel: Influencia de los Estados de Compresión Previa en la Resistencia a Tracción del Hormigón. A.T.E.P. (1975).

(12) SLate: A.C.I. Journal. (1967). 


\title{
Comentarios sobre factores desencadenantes y acelerantes de la corrosión de armaduras
}

\author{
C. ANDRADE (*)
}

\section{INTRODUCCION}

Se pretende con los presentes comentarios ofrecer unas reflexiones sobre la afirmación tan frecuentemente usada por los especialistas en corrosión de que "toda heterogeneidad es asiento de corrosión", es decir, que cualquier heterogeneidad puede dar lugar a la formación de dos zonas sobre el metal, ánodo y cátodo, que si encuentran continuidad eléctrica a través del metal e iónica a través del electrolito generarán una pila, que en última instancia va a producir la corrosión del metal por su disolución en el ánodo. Tal es el fundamento de la corrosión electroquímica.

La propia estructura de los metales en general, y del acero de construcción en particular, es de por sí heterogénea, como lo es, asimismo, el hormigón donde las armaduras se embeben, por lo que la consecuencia simplista, que se deduce cuando se oye la afirmación antes mencionada sin más matizaciones, es que lo extraordinario es que haya algún metal (de los no nobles) que logre mantenerse en estado no combinado, pues son innumerables las posibilidades de que existan heterogeneidades.

La primera y fundamental matización que es necesario hacer a tal axioma es que "toda heterogeneidad es asiento POTENCIAL de corrosión", y que en cada sistema particular metal-medio, habrá que establecer cuáles y en qué medida, son las circunstancias capaces de convertir en $A C T U A L$ el peligro potencial que supone la existencia de una heterogeneidad, o lo que es lo mismo, dado que las armaduras en un hormigón correctamente ejecutado se encuentran espontáneamente recubiertas por una capa de óxido adherente y libre de poros que las mantienen pasivadas, qué circunstancias pueden hacer pasar a las armaduras del estado pasivo, al activo o de corrosión.

Como no sólo la existencia de heterogeneidades es la responsable de la corrosión de armaduras, sino que también la presencia o ausencia de determinadas sustancias en el hormigón puede dar lugar a la oxidación del acero, en lo que sigue se hablará, en general, de "factores".

(*) C. Andrade. Dra. en Quimica Industrial. Dpto. de Química del IETCC. 


\section{DEFINICION DE FACTORES DESENCADENANTES Y ACELERANTES}

Se propone llamar factores DESENCADENANTES de la corrosión de armaduras a todos aquellos que sean capaces de generar sobre el metal zonas con una diferencia de potencial eléctrico tal, que la corriente promovida pueda romper la capa pasiva que recubre normalmente a las armaduras e iniciar así el proceso de oxidación del acero.

Ahora bien, el que una corrosión se desencadene porque las variables termodinámicas sean favorables para ello, no significa que progrese, y aquí se apunta la siguiente matización, es decir, no indica que la oxidación se vaya a producir con una velocidad apreciable. Aquellos factores o heterogeneidades que no son capaces de generar suficientes diferencias de potencial como para desencadenar el proceso de corrosión, pero que influyen sobre las variables cinéticas del mismo, haciendo que el proceso se desarrolle con apreciable velocidad, son los llamados factores ACELERANTES. Es obvio, que todos los factores desencadenantes son asimismo acelerantes, pero no se da la viceversa.

Añadir también que varios factores acelerantes, que individualmente no son capaces de romper la capa pasiva, pueden convertirse en desencadenantes si se dan simultáneamenie.

\subsection{Papel desempeñado por el oxígeno y la humedad}

Ambos factores juegan un papel esencial en el proceso de corrosión, por lo que aunque luego se hablará de ellos, aquí se quieren destacar unas cuestiones previas.

Sin la presencia simultánea en el hormigón de $\mathrm{O}_{2}$ y humedad, no es termodinámicamente posible la corrosión, y sin una cantidad mínima crítica, no es posible que se desarrolle con velocidad apreciable.

Ambos factores tienen un carácter ambivalente, pues cuando las armaduras están pasivas, una mayor cantidad de $\mathrm{O}_{2}$ y/o de humedad sirven para engrosar la capa pasivante y cuando las armaduras están activas corroyéndose, actúan intensificando notablemente el ataque.

Sobre la cantidad crítica de $\mathrm{O}_{2}$ que intensifica el ataque en las armaduras que se corroen, no existen datos cuantitativos, y sí, sin embargo, sobre la proporción de humedad relativa que, aproximadamente, es la misma que para el acero que se corroe espontáneamente en la atmósfera: alrededor del $60 \%$. Es decir, con HR $>60 \%$ se acentúan notablemente los procesos de corrosión que se hayan iniciado.

La situación más desfavorable para las armaduras, desde esta perspectiva analizada, es aquella en que el hormigón se encuentra en un medio ambiente con $\mathrm{HR}>60 \%$ o está parcialmente sumergido, y no aquellas en que se encuentra completamente saturado de agua o sumergido en ella, pues en estas circunstancias el $\mathrm{O}_{2}$ que puede llegar hasta la armadura, está limitado por el que previamente se pueda disolver en dicha agua. Si las armaduras no se corroen, entonces la humedad ambiental, sea cual sea, no altera su pasividad natural.

\section{ENUMERACION DE FACTORES DESENCADENANTES DE CORROSION}

Como el acero de las armaduras se encuentra pasivado, el tipo de corrosión que se puede manifestar es el LOCALIZADO: tanto por picaduras, como el propiamente localizado, como la corrosión fisurante bajo tensión. Ahora bien, si el $\mathrm{pH}$ del hormigón disminuye por una neutralización, entonces desaparece la capa pasiva y se manifiesta una corrosión generalizada. 
A continuación se pasará a enumerar y comentar los factores que, según juicio y experiencia de la autora y sin ánimo de ser exhaustiva, pueden ser considerados como desencadenantes y, posteriormente, los simplemente acelerantes, dividiéndolos según el tipo de corrosión que provocan.

\subsection{Corrosión localizada}

Este tipo de corrosión se caracteriza porque la capa pasiva sólo se rompe puntual (picaduras o fisuras) o parcialmente (ataque localizado). Estas zonas deterioradas actúan de ánodo frente al resto que permanece pasivo y actúa de cátodo, por lo que el ataque progresa en profundidad, pudiéndose llegar a la perforación del alambre.

Dadas las especiales peculiaridades del fenómeno conocido como "corrosión fisurante bajo tensión" se va a considerar en un apartado posterior.

Los factores que pueden ser considerados como desencadenantes de la corrosión por picaduras y la localizada son:

a) Dosis suficientes de iones despasivantes, en particular $\mathrm{Cl}^{-}$.

b) Pilas de $\mathrm{pH}$ o aireación diferencial.

c) Corrientes vagabundas.

d) Pilas galvánicas.

a) Los iones despasivantes son los más conocidos y peligrosos agentes desencadenantes de corrosión por picaduras. De entre los iones de este tipo que pueden estar presentes en la masa del hormigón, tanto porque se añadan como aditivos, como porque penetren desde el exterior (ambientes marinos, terrenos yesíferos, etc...), los responsables del mayor número de deterioros son los iones $\mathrm{Cl}^{-}$. En general son despasivantes todos aquellos iones de pequeño radio iónico que puedan formar compuestos solubles con el metal base y estén presentes en cantidades superiores a la llamada "crítica" o. "umbral".

En el caso de los $\mathrm{Cl}^{-}$, sobre la proporción crítica o umbral no hay acuerdo ni en las normas de los diversos países, ni entre los autores que lo han estudiado. Y esto por dos razones fundamentales: i) porque como los cloruros se combinan con los aluminatos que forman parte del cemento, dependerá de la proporción de éstos, del contenido de yeso, de la finura del cemento, así como de la edad del hormigón, la cantidad de ellos que estén en forma combinada o permanezcan "libres", siendo estos últimos los únicos agresivos, y ii) porque la agresividad depende fuertemente del contenido en humedad del hormigón.

Hay autores que señalan que un $2 \%$ de $\mathrm{Cl}_{2} \mathrm{Ca}$ en un hormigón compacto de cemento Portland puro no es dañino, y otros, que aluden a serios deterioros con proporciones del 1 ó incluso del 0,5\% (en relación al peso de cemento siempre).

Otros iones potencialmente despasivantes son $\operatorname{los} \mathrm{SO}_{4}{ }^{\circ}$ y los $\mathrm{S}^{=}$. Los primeros están presentes en el hormigón debido al yeso que se añade como regulador de fraguado, y los segundos aparecen en el cemento də escorias. Ambos, en las proporciones en que habitualmente están presentes en el hormigón, no son agentes desencadenantes. Los $\mathrm{SO}_{4}{ }^{2}$ pueden serlo cuando la estructura sufra un ataque por ellos y penetren en cantidad elevada hasta la armadura. Sobre la cantidad crítica de sulfatos que desencadena la corrosión de armaduras, se puede decir algo similar a lo mencionado para los cloruros en cuanto a la influencia de factores como: tipo de cemento, finura, etcétera. 
b) Circunstancias que provoquen pilas de $\mathrm{pH}$ y/o aireación diferencial son asimismo factores desencadenantes de corrosión de armaduras.

Estas pilas pueden estar provocadas por la presencia de fisuras de anchura suficiente, cuyos bordes además se carbonatan. $O$ cuando una estructura está parcialmente sumergida o enterrada (efecto de la "línea de agua"). O cuando existan sobre las armaduras espesores de recubrimiento de permeabilidad o grosor variable. Estas circunstancias hacen que la zona con menor $\mathrm{pH}$ o menor contenido en $\mathrm{O}_{2}$ se empiecen a corroer, actuando de ánodo frente al resto de la armadura que lo hará de cátodo.

c) Las corrientes vagabundas son también agentes desencadenantes de corrosión localizada, cuando se producen sobre una estructura con una cierta continuidad y durante períodos relativamente prolongados. En este caso actúa de ánodo la zona del acero por donde la corriente pasa del metal al medio, y de cátodo, por donde la corriente entra en el metal, es decir, por donde pasa del medio al acero.

Dado que el hormigón es un material muy poco conductor, este tipo de factor desencadenante es poco frecuente, pues la corriente, cuando existe, suele elegir en su mayor parte caminos más conductores.

d) La formación de pilas galvánicas, es decir, el contacto eléctrico entre el acero y otro metal de potencial electroquímico estándar muy diferente (Al ó Zn generalmente), tampoco es frecuente en el hormigón. Si bien, cuando se produce el deterioro suele ser muy rápido, por las elevadas intensidades galvánicas (de corrosión) generadas, actuando de ánodo el metal más activo con respecto a la serie de potenciales normales o estándar.

\subsection{Corrosión fisurante bajo tensión}

Se caracteriza por la rapidez y brusquedad con que se producen los accidentes. Para que aparezca se tienen que conjugar un metal susceptible de sufrir este tipo de ataque, propiedad que tiene el acero de alta resistencia, junto con una elevada tensión mecánica y un medio agresivo específico, que en el presente caso son los iones $\mathrm{OH}^{-}$.

En la superficie del metal se incuba una micro-grieta que progresa en profundidad por disolución del metal y concentración de las tensiones mecánicas en ella, produciéndose finalmente un desgarro brusco que lleva a la rotura sin estricción del acero cuando la grieta ha alcanzado una profundidad crítica. Todo ello agravado por el relativamente pequeño diámetro de los alambres de pretensado.

La corrosión bajo tensión (CBT) de los aceros para pretensado está gobernada por una serie de variables no bien conocidas ni delimitadas. Los diversos autores que la han estudiado llegan a conclusiones divergentes en cuanto a los mecanismos que gobiernan tanto la incubación de las fisuras como su propagación, todo ello agravado por el hecho de que, hasta el momento, no existe ningún método que mida la susceptibilidad del acero de alta resistencia a la CBT sobre alambres embebidos directamente en hormigón, por lo que los datos que se poseen se refieren al metal ensayado en condiciones supuestamente similares: disolución saturada de $\mathrm{Ca}(\mathrm{OH})_{2}$, solución hirviente de $\mathrm{NaOH}$, sulfocianuro amónico, etc.

Además de que el alambre esté sometido a una tensión por encima de un cierto valor crítico, que se suele expresar en función del \% de la carga de rotura, los factores que con los conocimientos actuales se suponen desencadenantes de este tipo de corrosión, son: 
a) Defectos o inclusiones superficiales en el acero.

b) Constitución metalográfica del acero.

c) Presencia de iones despasivantes, especialmente $\mathrm{Cl}^{-}$y $\mathrm{S}^{=}$.

d) Corrosión generalizada.

a) Raspaduras, entallas e inclusiones no metálicas sobre la superficie del acero parecen ser determinantes en la incubación de las microgrietas. En estas zonas la capa pasivante se encuentra distorsionada por lo que puede romperse con más facilidad al poner en tensión el acero. Se puede establecer así una diferencia de potencial suficiente entre la capa de óxido y la pequeña zona de metal desnudo, que al coincidir con un punto singular de la superficie: inclusión, etc..., puede provocar el comienzo de la disolución del metal.

b) A la estructura metalográfica del acero, adquirida mediante diversos tratamientos térmicos, también se le atribuye una influencia considerable. Así, es clásico ya el conocimiento de que los aceros templados y revenidos son mucho más susceptibles a este tipo de ataque que los patentados y trefilados. En la actualidad, diversos autores realizan estudios para establecer tiempos óptimos de tratamiento de envejecimiento artificial y de estabilizado, que minimicen la susceptibilidad del material a los fenómenos de CBT.

c) Ya se ha aludido a la propiedad que poseen los iones llamados despasivantes, especialmente cloruros y sulfuros en el caso de los cementos, de perforar la capa de pasividad, por lo que cuando el acero está sometido a tensiones elevadas, la presencia de estos iones puede provocar la rotura del alambre en tiempos mucho más cortos que cuando la tensión es pequeña o no existe.

d) Debido a que muchas veces se ha podido observar una cierta corrosión generalizada alrededor de la zona donde se han incubado las grietas, se piensa que esta circunstancia puede dar lugar a la CBT cuando se dan simultáneamente el resto de los requisitos. Es decir, la carbonatación, o pilas de $\mathrm{pH}$ o aireación diferencial, que puedan estimular la desaparición de la capa pasiva en una zona más o menos amplia, son consideradas por algunos autores como responsables del desencadenamiento de fenómenos de CBT en aceros tesos.

\subsection{Corrosión generalizada}

Este tipo de corrosión se caracteriza porque el metal se disuelve por igual, homogéneamente, en toda su superficie, por formación de multitud de micropilas.

Así como en la corrosión localizada, el riesgo de pérdida de sus características mecánicas lo presentaba el acero, en este caso el más afectado puede ser el hormigón, y según el grado de deterioro alcanzado, la adherencia metal-hormigón, pues como los productos de corrosión del acero ocupan alrededor de tres veces más volumen que las sustancias de partida, la aparición de tales óxidos en toda la superficie del metal ejerce un empuje tal sobre el hormigón que acaba por fisurarlo.

Para que este tipo de corrosión aparezca en las armaduras tiene que haberse producido previamente un descenso del $\mathrm{pH}$ habitual del cemento, de forma que el acero pase de la zona de pasividad, a la zona de corrosión de los diagramas de Pourbaix.

Un descenso tal de $\mathrm{pH}$ aparece por la acción continuada de sustancias ácidas sobre el hormigón, como pueden ser aguas carbónicas $\mathrm{D}$ simplemente ácidas, por el $\mathrm{CO}_{2}$ atmosféri- 
co, o por un efecto de lixiviación del $\mathrm{Ca}(\mathrm{OH})_{2}$ debido a la acción continuada de aguas muy puras.

El factor desencadenante de corrosión generalizada en las armaduras más frecuente es el debido a la carbonatación de la pasta por reacción con el $\mathrm{CO}_{2}$ atmosférico.

\section{FACTORES ACELERANTES DE TODOS LOS TIPOS DE CORROSION}

Se definieron como factores simplemente acelerantes a todos aquellos que por sí mismos, individualmente, no son capaces de generar suficientes diferencias de potencial como para que se produzca puntual, parcial o totalmente la rotura de la capa pasivante, pero que una vez rota ésta, son capaces de acelerar e intensificar el proceso corrosivo.

Los factores acelerantes por excelencia son la presencia de unas ciertas cantidades mínimas de $\mathrm{O}_{2}$ y humedad, como ya se expresó anteriormente, de tal forma que, una corrosión iniciada por la adición de $\mathrm{Cl}^{-}$al hormigón, puede pararse totalmente si el hormigón se seca en una atmósfera con HR alrededor del $50 \%$.

Son también factores acelerantes de todos los tipos de ataque los siguientes:

a) La presencia de adiciones activas en los cementos. Las adiciones activas (escorias y puzolanas) que se añaden a los cementos tienen la propiedad, entre otras muchas de las que exhiben, de reducir la reserva alcalina de los cementos, aunque mantienen el $\mathrm{pH}$ de la fase líquida, por lo menos en hormigones jóvenes, en los límites habituales entre 12 y 13 . Como los $\mathrm{pH}$ se mantienen, los aceros embebidos en hormigones fabricados con estos tipos de cementos se conservan protegidos indefinidamente al igual que los cementos Portland puros. Pero cuando la corrosión se desencadena por cualquiera de las circunstancias mencionadas anteriormente: presencia de $\mathrm{Cl}^{-}$, carbonatación, etc... el deterioro progresa a velocidades de 2 a 10 veces superiores que con cementos Portland puros, circunstancia que se achaca a la menor reserva alcalina de los cementos de adición al ser menos capaces de regenerar la capa pasiva alterada.

b) El cemento aluminoso es asimismo menos protector de las armaduras que los cementos Portland puros, debido a sus especiales características de no contener reserva alcalina similar a la de los Portland y además por exhibir un $\mathrm{pH}$ menor. Los aceros embebidos en hormigón de cemento aluminoso, cuando éste permanece inalterado se conservan protegidos indefinidamente, pues su pH es todavía el suficiente para situar el acero en la zona de pasividad. Pero cuando la corrosión comienza por otras circunstancias, también se produce a velocidades muy superiores de las que ocurrirían en los Portland.

c) La presencia de dos cementos de diferente tipo en contacto con una misma estructura, puede llegar a ser también un factor acelerante de la corrosión ya iniciada.

A este respecto, es necesario mencionar que las normas españolas EH-73 y EP-77 prohiben el empleo de dos cementos diferentes en contacto con una misma estructura, por considerar esta situación como corrosiva para el acero, lo que indica la creencia de que existe una relación biunivoca heterogeneidad-corrosión, pues el empleo de dos cementos diferentes es una aparente clara visualización del concepto heterogeneidad. Pero el uso de un cemento Portland puro y un P-Y que tienen similar pH y similar reserva alcalina, no debe generar sobre la armadura ninguna diferencia de potencial que signif:que el comienzo del ataque, ni tampoco el empleo de un Portland puro y un cemento de adición que tienen asimismo un $\mathrm{pH}$ muy pareci- 
do. Ahora bien, cuando la corrosión comienza por otras circunstancias, las diferencias de reserva alcalina en este último caso aludido, pueden sumarse a los procesos iniciados e intensificar el deterioro.

d) El tipo de acero utilizado puede suponer que un ataque se produzca con mayor o menor intensidad. Así, los aceros deformados en frío se deterioran más rápidamente que los de dureza natural, y los de alta resistencia más que los dos anteriores. Asimismo el acero corrugado se corroe más deprisa que el acero liso, si bien las diferencias absolutas entre todos ellos son pequeñas.

e) Todos los factores desencadenantes de corrosión de armaduras pero en proporciones menores de la crítica para desencadenar el proceso, son agentes acelerantes de otros ya iniciados. Así, un ataque por $\mathrm{Cl}^{-}$puede intensificarse en un hormigón ligeramente carbonatado, o una CBT, se produce mucho más deprisa si hay ligeras cantidades de $\mathrm{Cl}^{-}$presentes.

Finalmente, añadir que la concurrencia simultánea de varios agentes simplemente acelerantes pueden dar lugar a un factor desencadenante. Si se suman los efectos de todos ellos en una misma dirección, pueden aparecer diferencias de potencial suficientes para romper la capa pasiva. 